CONCISE REPORT

\title{
Toll-like receptor 4 gene polymorphisms and susceptibility to juvenile idiopathic arthritis
}

R Lamb, E Zeggini, W Thomson, BSPAR, R Donn

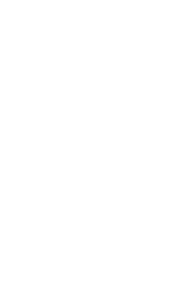

Ann Rheum Dis 2005;64:767-769. doi: 10.1136/ard.2004.026930

Objectives: To determine if polymorphisms within the Tolllike receptor 4 (TLR4) gene are associated and linked with juvenile idiopathic arthritis (JIA). To investigate any possible gene-gene (epistatic) interaction between TLR4 and macrophage migration inhibitory factor (MIF) gene polymorphisms. Methods: 313 simplex families (each containing one affected JIA proband) were genotyped. Two known functionally important single nucleotide polymorphisms (SNPs) within the TLR4 gene (Asp299Gly and Thr399lle) were typed by SNaPshot ddNTP primer extension and capillary electrophoresis.

Single point and multipoint transmission disequilibrium tests (TDT) were carried out through the extended TDT and TDT phase packages for the two TLR4 SNPs. Epistatic interaction between TLR4 haplotypes and the previously JIA associated MIF CAT - MIF- $173^{*} \mathrm{C}$ promoter haplotype was investigated by $\chi^{2}$ test and unconditional logistic regression in Stata version 7 .

Results: No distortion from random inheritance was observed by single point analysis for TLR4 Asp299Gly $(p=0.89)$ or TLR4 Thr399lle $(p=0.40)$. Similarly, no distortion in transmission was seen when the TLR4 haplotypes were studied $(p=0.54)$. Additionally, no evidence for genegene interaction between TLR4 polymorphisms and the previously associated MIF gene polymorphisms was found $(p=0.40)$.

Conclusions: No linkage or association was seen for Asp299Gly or Thr399lle SNPs of TLR4 with JIA susceptibility. No evidence of an epistatic interaction between these TLR4 polymorphisms and MIF polymorphisms was found.

J uvenile idiopathic arthritis (JIA) is a complex, clinically heterogeneous group of arthritides, which present before the age of 16 years. We have recently shown, in two separate cohorts, that the macrophage migration inhibitory factor (MIF), a proinflammatory cytokine, is both linked and associated with JIA. ${ }^{12}$ A relationship between MIF and Tolllike receptor 4 (TLR4) was found in a study of MIF deficient mice, which were resistant to endotoxic shock. MIF deficient macrophages were hyporesponsive to lipopolysaccharide (LPS) and to Gram negative bacteria owing to a down regulation of TLR4. ${ }^{3}$ TLR4 is involved in the adaptive and innate immune responses by binding to pathogens, microbial toxins, or endogenous ligands such as LPS, heat shock proteins, fragments of hyaluronic acid, and fibronectin. Formation of TLR-ligand complexes activates signal transduction pathways and the transcription of immune genes, resulting in the release of inflammatory mediators (reviewed by Heine and Lien $^{4}$ ). $T$ cell reactivity to human heat shock protein 60 has been shown in oligoarticular JIA, and activated $\mathrm{T}$ cells have been found to be regulatory and contribute to disease remission. ${ }^{5}$ Furthermore, TLR4 knockout mice have been reported to have significantly reduced joint swelling and cell influx compared with wild-type mice. ${ }^{6}$ These observations supports the hypothesis that TLR4 is a candidate gene for investigation in JIA, although the mechanism by which TLR4 may contribute to JIA pathology has yet to be fully determined.

Two single nucleotide polymorphisms (SNPs) of TLR4 have been described previously, ${ }^{7}$ an amino acid (aa) change at aa 299 of aspartic acid to glycine (Asp299Gly) and a mis-sense mutation replacing threonine with isoleucine at aa 399 (Thr399Ile). These polymorphisms are associated with a blunted response to inhaled LPS in humans and, furthermore, the Asp299Gly interrupts TLR4 mediated LPS signalling. ${ }^{7}$

We have investigated the Asp299Gly and the Thr399Ile TLR4 polymorphisms in a cohort of JIA probands and their parents. Positive linkage and association with the $\mathrm{CATT}_{7}$ MIF- $173^{*} \mathrm{C}$ haplotype has previously been described in this cohort. ${ }^{2}$ As a biological pathway relating MIF and TLR4 has been detailed, ${ }^{3}$ it is possible that a combination of particular polymorphisms of these two genes might lead to a manifold increase in the risk of JIA susceptibility. For this reason we looked for any such epistatic relationship between MIF and the TLR4.

\section{PATIENTS AND METHODS}

Blood samples were obtained with informed written consent. Ethical approval was obtained from MREC (99/8/84) and the University of Manchester committee on the ethics of research on human beings (8/92/(i) (b)). Three hundred and thirteen UK white simplex families (each simplex family contained one affected JIA proband and healthy parent(s)) from the British Society of Paediatric and Adolescent Rheumatology (BSPAR) national repository for JIA were genotyped for the two TLR4 SNPs Asp299Gly and Thr399Ile (with reference to Genbank Accession No U93091). All patients with JIA had been classified according to the ILAR classification criteria. Table 1 shows the number of patients with JIA in each ILAR subgroup. Genotyping was by SnaPshot ddNTP primer extension and capillary electrophoresis as previously described. ${ }^{1}$ Briefly, polymerase chain reactions (10 $\mu \mathrm{l}$ final volume) contained $10 \mathrm{ng}$ of genomic DNA, $0.4 \mu \mathrm{l}$ of each primer $(25 \mathrm{pmol} / \mu \mathrm{l}), \mathrm{l} \mu \mathrm{l}$ of dNTPs (Bioline, $2 \mathrm{mM}), 0.3 \mu \mathrm{l}$ of $\mathrm{MgCl}_{2}$ (Bioline, $1.5 \mathrm{mM}$ ), $1 \mu \mathrm{l}$ of $\mathrm{NH}_{4}$ buffer (Bioline, $10 \times$ ), and $0.2 \mu \mathrm{l}$ of Taq polymerase (Bioline, $5 \mathrm{U} / \mu \mathrm{l}$ ) using the following primers and probes, all written 5'-3: 299 forward primer CCA TTG AAG AAT TCC GAT TAG CAT, 299 reverse primer GGG AAA ATG AAG AAA CAT TTG TCA A, 299 probe AAT TAA ATA AGT CAA TAA TA, 399 forward primer TCT

Abbreviations: aa, amino acid; JIA, juvenile idiopathic arthritis; LPS, lipopolysaccharide; MIF, migration inhibitory factor; SNPs, single nucleotide polymorphisms; TDT, transmission disequilibrium test; TLR4, Toll-like receptor 4 


\begin{tabular}{|lc|}
\hline Table 1 JIA probands by ILAR subgroup \\
\hline ILAR Group & $\begin{array}{c}\text { Number of JIA } \\
\text { families }\end{array}$ \\
\hline Systemic arthritis & 50 \\
Persistent oligoarthritis & 84 \\
Extended oligoarthritis & 46 \\
RF* negative polyarthritis & 57 \\
RF positive polyarthritis & 8 \\
Enthesitis related arthritis & 22 \\
Psoriatic arthritis & 20 \\
Unclassifiable & 26 \\
\hline *RF, rheumatoid factor & \\
Classification according to ILAR criteria. \\
\hline
\end{tabular}

CAG TAG AAA TGG CTT GAG, 399 reverse primer CTC ACT CAT TTG TTT CAA ATT G, and 399 probe AGC TCA GAT CTA AAT ACT TTA GGC TG.

The fluorescent peaks obtained were detected with the use of the Genescan 3.7 analysis software package. The Genotyper 3.7 software package was then used to assign allele names.

\section{Statistical methods}

Deviation from Hardy-Weinberg equilibrium was tested using Stata version 7 (Stata Corporation, College Station, Texas, USA). The extended transmission disequilibrium test (TDT $)^{9}$ that detects linkage and association was performed for the two TLR4 SNPs Asp299Gly and Thr399Ile. Empirical $\mathrm{p}$ values were obtained by running 10000 Monte Carlo simulations. This study had $80 \%$ power to detect, at a single locus, an odds ratio of 2 at the 0.05 significance level (Quanto version 0.4). Linkage disequilibrium between the SNPs was calculated using HelixTree (GoldenHelix, Inc, Montana, USA). Multipoint linkage and association for the TLR4 Asp299Gly and Thr399Ile haplotypes were investigated through the TDT phase programme, which tests for the distortion of transmission of haplotypes from parents to affected offspring. ${ }^{10}$ The case-only design was employed to estimate gene-gene interaction effects between MIF and TLR4. ${ }^{11}$ The TLR4 haplotypes and the previously associated MIF CATT $_{7}$-MIF- $173^{*} \mathrm{C}$ promoter haplotype were estimated and assigned to affected subjects through snphap (David Clayton, Cambridge, UK). This study had $80 \%$ power to detect a gene-gene interaction conferring a relative risk of at least 4 (Quanto version 0.4). ${ }^{12}$ The presence of an interactive effect was tested through a standard $\chi^{2}$ test and through unconditional logistic regression in Stata version 7.

\section{RESULTS}

The minor allele frequencies in patients with JIA for the two SNPs of TLR4 Asp299Gly and Thr399Ile were 5\% and 6\%, respectively. No significant deviation from Hardy-Weinberg equilibrium was observed for either of the TLR4 SNPs (Asp299Gly, $\mathrm{p}=1$; Thr399Ile, $\mathrm{p}=0.61$ ). Single point analysis using the extended TDT showed no distortion from random inheritance for either the TLR4 Asp299Gly or the Thr399Ile SNP. Non-significant empirical $p$ values were observed for the TLR4 Asp299Gly $(p=0.89)$ and the Thr399Ile $(p=0.40)$ SNPs.

Strong linkage disequilibrium was observed between the two SNPs in the patients with JIA, with a $\mathrm{D}^{\prime}$ value of 0.88 ( $\mathrm{D}^{\prime}$ values can range from 0 to 1 , reflecting no and complete linkage disequilibrium, respectively).

However, no significant distortion in transmission was seen for the TLR4 haplotypes (empirical $p=0.54$ ). The results show that these two TLR4 SNPs are not linked or associated with JIA as a whole. Furthermore, subgroup-specific analysis showed no linkage and association with either of the TLR4 SNPs, or their haplotypes. However, the power to detect an effect was limited owing to the small number of informative transmissions when individual subgroups were considered.

No evidence for an epistatic effect between TLR4 haplotypes and the associated MIF promoter haplotype $\left(\mathrm{CATT}_{7^{-}}\right.$ MIF- $\left.173^{*} \mathrm{C}\right)$ was seen $(\mathrm{p}=0.40)$.

\section{DISCUSSION}

Only a limited number of genetic associations with JIA have stood the test of replication. MIF is one such locus. Promoter polymorphisms of MIF have now been found to be linked and associated with JIA susceptibility in two independent disease cohorts. ${ }^{12}$ The mechanism by which MIF acts as a susceptibility locus has not yet been determined (reviewed by Calandra and $\operatorname{Roger}^{13}$ ). Clearly MIF has multiple actions that might be contributory to its role in increasing risk of JIA. However, we were interested to pursue further the known observation that MIF regulates immune responses by modulating the expression of TLR4. ${ }^{3}{ }^{14}$ TLR4 can recognise a broad variety of pathogen associated molecular patterns (PAMPs) including LPS. ${ }^{4}$ Subsequent to the binding of such PAMPs, the innate immune response is activated. Signalling by means of nuclear factor $\kappa \mathrm{B}(\mathrm{NF \kappa B})$ releases several cytokines, including tumour necrosis factor $\alpha$ and interleukin $1 \beta$ and matrix metalloproteinases, resulting in inflammation. In addition, TLR4 has recently been shown to have a role in dendritic cell maturation and initiation of the adaptive immune response. ${ }^{15}$ Polymorphisms of the TLR4 gene may therefore themselves, or in combination with MIF polymorphisms, contribute to increased risk of JIA.

Arbour et al described two TLR4 SNPs (Asp299Gly and Thr399Ile) and showed that they were associated with a blunted response to inhaled LPS. ${ }^{7}$ Smirnova et al used sequencing to identify potential polymorphisms of TLR4. Their screen included all of the TLR4 coding sequence (exons 1-3) (in 141 white subjects) and $1.1 \mathrm{~kb}$ of intron 2 (studied in 50 white subjects). Only the TLR4 Asp299Gly and Thr399Ile were seen at frequencies of $>1 \%{ }^{8}$ We therefore studied the two TLR4 SNPs, Asp299Gly and Thr399Ile in a large sample of JIA simplex families, all of which had previously been characterised for MIF gene polymorphisms.

No association with either the Asp299Gly or Thr399Ile TLR4 SNP was seen in our total JIA population. These SNPs are in very strong linkage disequilibrium $\left(\mathrm{D}^{\prime}=0.88\right)$. We found no evidence for altered transmission of TLR4 haplotypes from unaffected parents to a child affected with JIA. Our study lacked sufficient power to demonstrate any JIA subgroup-specific TLR4 effect.

Finally, we wished to explore the possibility that a biologically plausible interaction between TLR4 and MIF resulted in gene-gene interaction and a subsequently increased risk of disease. Our study had sufficient power to detect a gene-gene interaction conferring a relative risk of 4 but had only limited power to detect more modest relative risks. Following the case-only model for gene-gene interaction, no evidence for such an interactive relationship and JIA susceptibility was seen. This study used the previously

\section{Key messages}

- The Asp299Gly and Thr399lle SNPs of the TLR4 gene are not linked or associated with JIA susceptibility.

- No evidence exists for gene-gene interaction of TLR4 and MIF with JIA susceptibility. 
identified SNPs within TLR4. We did not perform de novo coding region mutation screening of patients with JIA, nor did we attempt to identify intronic or promoter region SNPs of TLR4 for use in our study. It remains possible, therefore, that discrete JIA TLR4 changes exist, or that as yet uncharacterised intronic or promoter TLR4 SNPs are important in conferring increased risk of JIA. However, this would only be the case if such additional SNPs were not in linkage disequilibrium with either the TLR4 Asp299Gly or Thr399Ile SNP that we have investigated. Overall, we have not observed any evidence for involvement of the TLR4 Asp299Gly or Thr399Ile polymorphisms with JIA susceptibility.

\section{ACKNOWLEDGEMENTS}

This work was funded by the ARC, UK. Rebecca Lamb is funded by an MRC studentship.

\section{Authors' affiliations \\ R Lamb, W Thomson, R Donn, Arthritis Research Campaign's} Epidemiology Unit (ARC/EU), University of Manchester, Stopford Building, Oxford Road, Manchester M13 9PT, UK

E Zeggini, Centre for Integrated Genomic Medical Research, University of Manchester, Stopford Building, Oxford Road, Manchester M13 9PT, UK

R Donn, Centre for Molecular Medicine, University of Manchester, Stopford Building, Oxford Road, Manchester M13 9PT, UK

Contributors to The British Society of Paediatric and Adolescent Rheumatology (BSPAR) are: $\operatorname{Dr} M$ Abinun, $\operatorname{Dr} M$ Becker, $\operatorname{Dr} A$ Bell, Professor A Craft, Dr E Crawley, Dr J David, Dr H Foster, Dr J GardenerMedwin, Dr J Griffin, Dr A Hall, Dr M Hall, Dr A Herrick, Dr P Hollingworth, $\operatorname{Dr} L$ Holt, $\operatorname{Dr} S$ Jones, $\operatorname{Dr} G$ Pountain, $\operatorname{Dr} C$ Ryder, Professor T Southwood, Dr I Stewart, Dr H Venning, L Wedderburn, Professor P Woo, Dr S Wyatt

Correspondence to: Miss R M Lamb; rebecca.lamb@man.ac.uk

Accepted 10 September 2004

Published Online First 21 October 2004

\section{REFERENCES}

1 Donn R, Alourfi Z, De Benedetti F, Meazza C, Zeggini E, Lunt M, et al. Mutation screening of the macrophage migration inhibitory factor gene: positive association of a functional polymorphism of macrophage migration inhibitory factor with juvenile idiopathic arthritis. Arthritis Rheum 2002;46:2402-9

2 Donn RP, Alourfi Z, Zeggini E, Lamb R, Jury F, Lunt M, et al. A functional promoter haplotype of macrophage migration inhibitory factor (MIF) is linked and associated with juvenile idiopathic arthritis. Arthritis Rheum 2004;50:1604-10.

3 Roger T, David J, Glauser MP, Calandra T. MIF regulates innate immune responses through modulation of Toll-like receptor 4 . Nature 2001:414:920-4.

4 Heine $\mathbf{H}$, Lien $\mathrm{E}$. Toll-like receptors and their function in innate and adaptive immunity. Int Arch Allergy Immunol 2003;130:180-92.

5 de Kleer IM, Kamphuis SM, Rijkers GT, Scholtens L, Gordon G, de Jager W, et al. The spontaneous remission of juvenile idiopathic arthritis is characterized by $C D 30+T$ cells directed to human heat-shock protein 60 capable of producing the regulatory cytokine interleukin-10. Arthritis Rheum 2003;48:2001-10.

6 Radstake TR, Franke B, Hanssen S, Netea MG, Welsing P, Barrera P, et al. The Toll-like receptor 4 Asp299Gly functional variant is associated with decreased rheumatoid arthritis disease susceptibility but does not influence disease severity and/or outcome. Arthritis Rheum 2004;50:999-1001.

7 Arbour NC, Lorenz E, Schutte BC, Zabner J, Kline JN, Jones M, et al. TLR4 mutations are associated with endotoxin hyporesponsiveness in humans. Nat Genet 2000;25:187-91.

8 Smirnova I, Hamblin MT, McBride C, Beutler B, Di Rienzo A. Excess of rare amino acid polymorphisms in the Toll-like receptor 4 in humans. Genetics 2001; 158:1657-64.

9 Sham PC, Curtis D. An extended transmission/disequilibrium test (TDT) for multi-allele marker loci. Ann Hum Genet 1995;59:323-36.

10 Dudbridge F, Koeleman BP, Todd JA, Clayton DG. Unbiased application of the transmission/disequilibrium test to multilocus haplotypes. Am J Hum Genet 2000;66:2009-12.

11 Yang Q, Khoury MJ, Sun F, Flanders WD. Case-only design to measure genegene interaction. Epidemiology 1999;10:167-70.

12 Gauderman WJ. Sample size requirements for association studies of genegene interaction. Am J Epidemiol 2002;155:478-84.

13 Calandra T, Roger T. Macrophage migration inhibitory factor: a regulator of innate immunity. Nat Rev Immunol 2003;3:791-800.

14 Calandra T, Froidevaux C, Martin C, Roger T. Macrophage migration inhibitory factor and host innate immune defenses against bacterial sepsis. $J$ Infect Dis 2003;187(suppl 2):S385-90.

15 Dabbagh K, Dahl ME, Stepick-Biek P, Lewis DB. Toll-like receptor 4 is required for optimal development of Th2 immune responses: role of dendritic cells. $\mathrm{J}$ Immunol 2002;168:4524-30. 